\title{
3D Mapping and Characterization of Sistema Zacatón from DEPTHX (DEep Phreatic THermal explorer)
}

\author{
Marcus Gary ${ }^{1,4}$, Nathaniel Fairfield ${ }^{2}$, William C. Stone ${ }^{3}$, \\ David Wettergreen ${ }^{2}$, George Kantor ${ }^{2}$, John M. Sharp, Jr. ${ }^{4}$
}

\begin{abstract}
Underwater exploration at Sistema Zacatón reached human limits when Jim Bowden descended to a depth of 289 meters April 6, 1994. In order to understand this karst system, unmanned robotic exploration is required to document the immense geometry of the underwater caves. The DEPTHX (DEep Phreatic THermal eXplorer) vehicle was developed with support from NASA to approach this problem, with the additional impetus to address robotic exploration in outer space. During the winter and spring of 2007, DEPTHX conducted three-dimensional (3D) underwater mapping missions of 4 cenotes in Sistema Zacatón: El Zacatón, Caracol, Poza Verde, and La Pilita. The detailed maps discovered no lateral tunnels connecting the cenotes, which are only 100-200 meters apart from each other. The deepest cenote, El Zacatón was bottomed out at a depth of 319 meters (339 m including above water cliff), making it the deepest underwater vertical shaft and second deepest underwater cave in the world. No side tunnels were discovered. However, the 3D data revealed geomorphic features of the cenotes to document how the karst system may have evolved through time. Spatial geochemical data collected contemporaneously during mapping missions indicate that water in the three warmest cenotes is extremely homogeneous, and the cooler cenote displays chemoclines similar to lacustrine type settings. The data collected by DEPTHX are being used with other types of geologic information to investigate the specific nature of hypogenic karstification that formed the cave system.
\end{abstract}

\section{INTRODUCTION}

Geologic investigations related to the origin of caves and karst features rely on accurate and detailed geographic information to convey the morphology, scale, physical characteristics, and chemical parameters that are essential for evaluating the geologic processes that developed the karst landscape. Sistema Zacatón, a unique karst area in the state of Tamaulipas Mexico (Figure 1), is hypothesized to have formed by hypogenic karst processes influenced by volcanic activity. It is characterized by deep phreatic shafts, travertine capped sinkholes, and ramiform vadose cave passages. (Gary and Sharp, 2006). The primary feature of the system, Él Zacatón, is the deepest underwater cave shaft in the world with a minimum depth of 319 meters (Gary et. al. 2003; Gary 2001).

\footnotetext{
${ }^{1}$ Zara Environmental, LLC, Buda, Texas, (512) 470-8029, marcus@zaraenvironmental.com

${ }^{2}$ Robotics Institute, Carnegie Mellon University, Pittsburgh, Pennsylvania

${ }^{3}$ Stone Aerospace, Del Valle, Texas

${ }^{4}$ Jackson School of Geosciences, The University of Texas at Austin
} 




FIG. 1. Aerial photograph of the southern portion of Sistema Zacatón showing the major cenotes imaged by the DEPTHX project: Zacatón, Caracol, Poza Verde, and La Pilita. The location of Sistema Zacatón is in northeastern Mexico (inset).

The ability to represent and characterize karst landscapes, including the interactions of all components, has not been fully achieved (White 2002), and new methods to quantify and document karst features are required. The high heterogeneity associated with karst areas creates challenges in developing a comprehensive understanding of karst systems (Bakalowicz 2005), and a geomorphological strategy for landscape characterization offers a framework for karst landscape assessment (Veni 1999). The karst landscape of Sistema Zacatón consists of a variety of karst features. Features vary from horizontal, dry cave passages to deep, phreatic shafts; surface features include dolines, cave entrances, springs, and sinking streams. While there has been significant progress with satellite or laser surveys of surface geography, the characterization of underwater geography has been very limited and laborious (an Ende, 2001). In this paper, we present results from the first autonomous underwater robotic exploration of a large-scale geological formation, Sistema Zacatón.

\section{Overview of DEPTHX Project}

The DEPTHX (DEep Phreatic THermal eXplorer) project was a three-year (20042007) NASA-funded effort with the primary objective of using an autonomous vehicle to explore and characterize the unique biology of the Sistema Zacatón cenotes. The robotic exploration and search for microbial life in Zacatón is an analog mission for the search for life in the liquid water ocean beneath the frozen surface of Europa. The DEPTHX vehicle (Figure 2) is a hovering autonomous underwater vehicle (AUV) designed to explore flooded caverns and tunnels and to collect environmental data and samples from the water column and cavern walls. During two field sessions in Mexico, the DEPTHX vehicle explored four cenotes, creating the first maps of these underwater structures and collecting precisely localized scientific samples for laboratory analysis. 

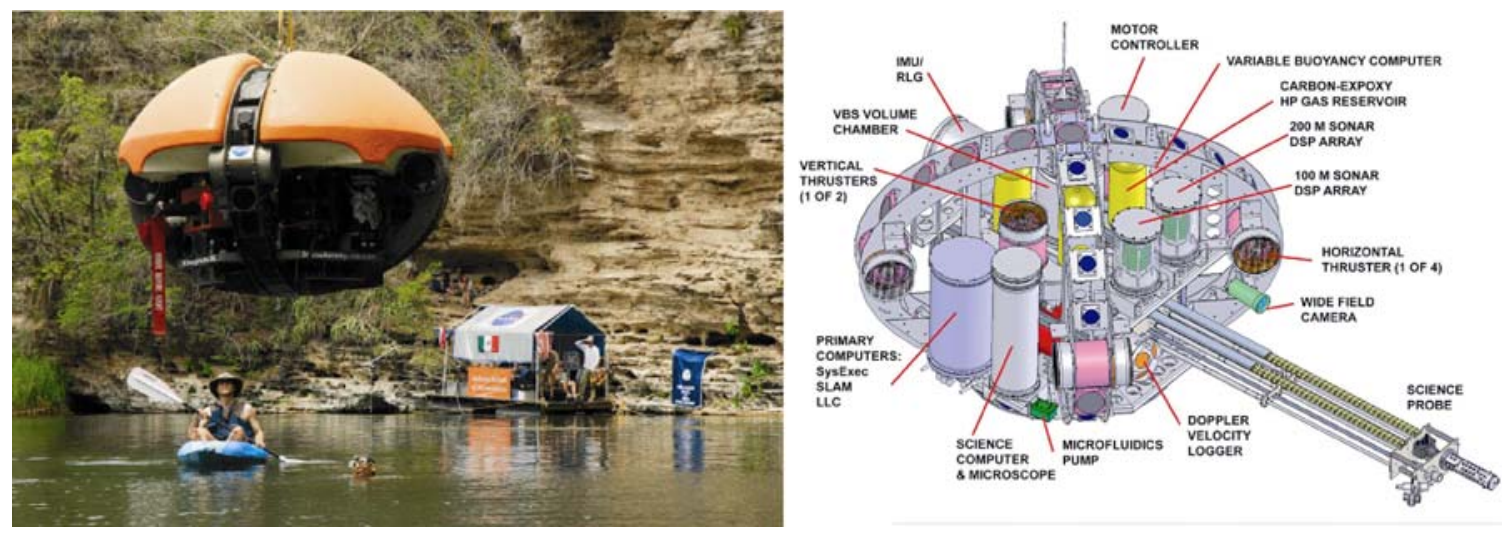

FIG. 2. Left photo shows the DEPTHX probe lowered into the cenote Zacatón. The right schematic shows primary components of DEPTHX. (Images from Stone Aerospace, 2007).

The DEPTHX vehicle has an ellipsoidal (oblate spheroid) shape that measures approximately 1.5 meters in height and 1.9 meters in both length and width. Its dry mass is $1500 \mathrm{~kg}$. Four large pieces of syntactic foam are mounted on the top half of the vehicle, passively stabilizing the vehicle roll and pitch. The vehicle can move directly in the remaining four degrees of freedom (forward, starboard, down, and heading) using six thrusters driven by brushless DC motors. The cruising speed of the vehicle is about 0.2 meters per second. The vehicle is powered by two 56-volt lithium-ion batteries with a total capacity of $6.2 \mathrm{KWh}$, enough to power the vehicle during a four-hour exploration mission.

The DEPTHX vehicle has a full suite of underwater navigation sensors, including a Honeywell HG2001AC inertial measurement unit (IMU), two Paroscientific Digiquartz depth sensors, and an RDI Navigator $600 \mathrm{kHz}$ Doppler velocity log (DVL). The specifications for the IMU are roll/pitch: $0.2^{\circ} 2 \sigma$, yaw: $0.4^{\circ} 2 \sigma$, for the (DVL) velocities $0.3 \mathrm{~cm} / \mathrm{s} 1 \sigma$, and for the depth sensors $0.01 \%$ of full range $(14 \mathrm{~cm}$ for our $1400 \mathrm{~m}$ rated sensor). The two depth sensors are tared, or zeroed, with respect to atmospheric pressure, at the start of each day. The DVL is mounted to the front of the vehicle facing forward and tilted down 30 degrees from horizontal, a nonstandard configuration for this instrument. The usual DVL configuration points straight down so that it can make Doppler velocity measurements sense the subaquatic floor with its four acoustic beams and lock on to surface location. In our application, it is difficult to predict the relative direction to surfaces useful for DVL lock. The top portion of Zacatón is a chimney with a diameter of approximately 80 meters (Fairfield et al., 2005), so the forward-looking configuration allows the DVL to lock on to one of the vertical walls in most situations. This configuration can cause the DVL to lose one or more beams bottom lock in more wide-open waters. Loss of the beam's bottom lock can also occur at extremely short ranges, or when passing over highly irregular terrain. A full description of the engineering development is described in Stone (2007).

The robotic systems of the DEPTHX vehicle have capabilities beyond only 3D map generation, although this was the primary data collection system. The great depth and volume of the cenotes of Sistema Zacatón make it practically impossible to document accurately the geometry of the karst features, so applying precise sonar (Sound 
Navigation And Ranging) measurement data from a robotic platform removed the hazards of direct human mapping at these depths. The maps were used to define exact volume and geomorphology of the caves. Once 3D maps were generated by the DEPTHX vehicle, other autonomous systems such as multi-parameter water quality meters, wall samplers, water samplers, and video/still cameras could collect data, and assign precise spatial information related to the data / samples collected. The information from all systems is used to document the present conditions and features of the cenotes and provide the analytical basis for hydrogeologic hypothesis related to their speleogenesis.

\section{METHODS}

\section{Acquisition of $3 D$ spatial data}

High-resolution digital mapping of Sistema Zacatón has two primary 3D methods integrated to capture the physical geometry of the caves and karst features. These methods provide a unique mechanism to visualize, analyze, and infer the specific physical expression of the karst system. Areas above the water table were imaged using a lidar (Light Distance and Ranging) instrument, and the phreatic zone of Zacatón was imaged using sonar technology that is the primary mapping system on the DEPTHX probe. Data collected from both of these methods have been georeferenced using a set of benchmarks precisely located by GPS (Global Positioning System) measurements. These benchmarks provide the common spatial context used to place the LIDAR and sonar data in a proper geographic Cartesian coordinate system.

Both the lidar and sonar data were georeferenced from benchmarks set using carrier phase-differential GPS measurements. Two Trimble 4000SSI receivers were used to measure the location of the benchmarks with a precision of $<2$ centimeters in the $\mathrm{X}-\mathrm{Y}$ plane (horizontal), and $<4$ centimeters in the $\mathrm{Z}$-direction (vertical). Benchmarks located in areas without a clear view of the sky were surveyed using a Leica Total Station instrument, and this instrument was also used to precisely locate the origin coordinate for underwater mapping missions by the DEPTHX vehicle.

Lidar data. Lidar imaging provides detailed 3D analyses of the cenotes at the land surface. Laser scanning has been used to image karst features in other locations such as Carlsbad Caverns, New Mexico (Nagihara et al., 2002) In January 2002, detailed scanning of the cenotes commenced at Zacatón, Caracol, La Pilita, Azufrosa, and the dry cave Caverna Los Cuarteles. Verde has not yet been surveyed with lidar because of the extremely dense vegetation surrounding the cenote. Preliminary lidar surveying provides a 3D image of the land and water surface associated with the cenotes. Advantages to using lidar, a ground-based, static mount similar to aerial platform-based lidar imagery, include imaging under tree canopies, manmade structures, or within overhung rock structures. Images were produced using a Riegl LPM98-VHS instrument, which operates in the near-infrared spectrum at 900 nanometers with a pulse rate of $1 \mathrm{kHz}$. A full scan will produce $2.6 \times 10^{6} 3 \mathrm{D}$ data points in about 90 minutes. Scan locations -- selected to achieve full feature coverage once registered -- were established directly above surface benchmarks described above. The lidar datasets are meshed to form a geo-registered point-cloud that represents surface features down to water level inside the cenotes (Gary 
and Stone, 2002).

SONAR data. For mapping, the DEPTHX vehicle has an array of 54 narrow-beam $\left(2^{\circ}\right.$ beam-width) sonars that provide a constellation of range measurements around the vehicle at about $1 \mathrm{~Hz}$ (Figure 2). This array is in the shape of three great circles, a configuration that was arrived at after studying the suitability of various sonar geometries for 3D mapping (Fairfield et al., 2005). The sonar hardware was originally developed to map Wakulla Springs, Florida, in 1998 with the Digital Wall Mapper (Stone et al., 2000), then modified and expanded for the DEPTHX vehicle. The sonars have long ranges (some $100 \mathrm{~m}$ and others $200 \mathrm{~m}$ ), and the accuracy of the range measurements is about $10 \mathrm{~cm}$ however, the low resolution, update rate, and point density make the mapping problem significantly more difficult than it is with ranging sensors like a laser scanner that provide fast, accurate, high-resolution ranges.

\section{Analysis and processing of spatial data}

Underwater localization and mapping. Building maps from autonomous underwater exploration presents two challenges. First, there is no absolute position information (like GPS), so the vehicle must perform its own localization estimate. Second, by the definition of exploration, there are no known reference points to provide ground truth for a map, except for the starting point. So the vehicle has to build a map with new data, and localize itself using that map at the same time. The approach we describe below builds highly accurate maps, but due to the non-unique nature of localization and mapping, it is susceptible to unavoidable error (or drift) over long distances, except when the vehicle can close loops, returning to previously mapped areas.

For DEPTHX we employ a probabilistic algorithm that provides a best estimate of the vehicle trajectory and a 3D map of the environment. Here, we focus on the 3D map representation, see (Fairfield et al. 2006; 2007) for a more complete description of our simultaneous localization and mapping (SLAM) approach.

3D Maps - Evidence Grids: Sonar measurements are noisy and unable to resolve fine features, but integrated over time they provide information about the geometry of the environment around the vehicle. We attempted to filter the sonar data, but due in equal parts to limitations of the sonar hardware and to the multipath noise in the cenote environment, we were unable to filter out the noisy ranges without discarding an unacceptable fraction of the good ranges as well. In order to combine the individual noisy sonar measurements probabilistically, we used a 3D evidence grid (Moravec and Elfes, 1985). An evidence grid is a uniform discretization of space into cells in which the value of each cell indicates the estimated probability that the cell is occupied by a wall, based on the sonar measurements. In 3D, the cells are cubes, or voxels. Because evidence grids fuse the occupancy information from multiple measurements, they converge to an accurate representation of the environment, even in the presence of many noisy ranges.

A measurement model is used to determine how a particular sonar measurement affects the map. We modeled the 56 individual sonars as producing 2-degree cones projecting from the vehicle. Given a particular range measurement, our model states that voxels within the cone are probably empty and voxels at the end of the cone are probably occupied. A modified Bresenham 3D ray tracing algorithm (Bresenham, 1965) is used to 
merge these probabilities with the information already contained in the $3 \mathrm{D}$ evidence grid, according to a Bayesian update rule.

A major drawback of the 3D evidence grid approach is that the memory required to store them increases as the cube of the size of the map. For reasonable map sizes and resolutions, the memory requirements quickly become intractable, especially considering that our particle filter SLAM approach requires multiple maps to represent different possible vehicle trajectories. To circumvent this storage and processing problem, we use the Deferred Reference Counting Octree data structure described by Fairfield et al. (2007). This data structure exploits shared regions between particle maps and efficiently represents sparse volumes, yielding a significant performance boost that allows us to represent maps that would not even fit into memory as a uniform array.

Merging lidar and sonar data. The maps produced by the SLAM system have no fixed point except for the starting point. The starting point was defined by a plumb bob that hung down to the surface of the water. Before initializing the SLAM system, the vehicle was driven to exactly below this plumb bob. In order to register this starting point in the world frame, we used a Total Station to measure its relative position from the geo-referenced benchmarks described above. Excepting a few record-keeping mistakes, this gave us an easy method for registering the lidar-based and sonar-based maps.

Generation of 3-D maps. Using the SLAM method described above, we built maps of the four cenotes of Sistema Zacatón. These maps were then transformed into three formats for human interpretation: point clouds, evidence grids, and triangle meshes. We generated point clouds by projecting the sonar beam end-points according to the SLAM trajectory of the vehicle. Point clouds are simple to manipulate, but there are many spurious points due to the noise of the underlying sonar ranges. The evidence grids are constructed as part of SLAM process and are much more robust to sonar noise. However, they can be difficult to visualize. However, we can extract a single occupancy iso-surface from the evidence grid using marching tetrahedra (Lorensen and Cline 1987) to create a mesh, which is both robust to noise and easy to visualize (Figure 3 ).

\section{Spatial geochemical data}

In order to characterize the basic geochemistry of cenotes in Sistema Zacatón, a HydroTech HT6 multiparameter meter was used to collect water temperature, $\mathrm{pH}$, specific conductance, dissolved oxygen, and oxygen redox potential at a frequency of 1 Hz. Data points exist for every location traveled by the DEPTHX vehicle during all mapping and science missions during the project. An additional sensor independent from the HydroTech unit measured electrical potential changes across a special electrode intended to relate to sulfide concentrations in the water, also at a frequency of $1 \mathrm{~Hz}$. This sensor, however, was unable to be properly calibrated at the environmental conditions experienced. Relative changes in voltages were measured. The DEPTHX vehicle was also equipped with water and rock (wall material) sampling hardware, which collected samples to depths of 293 meters. 

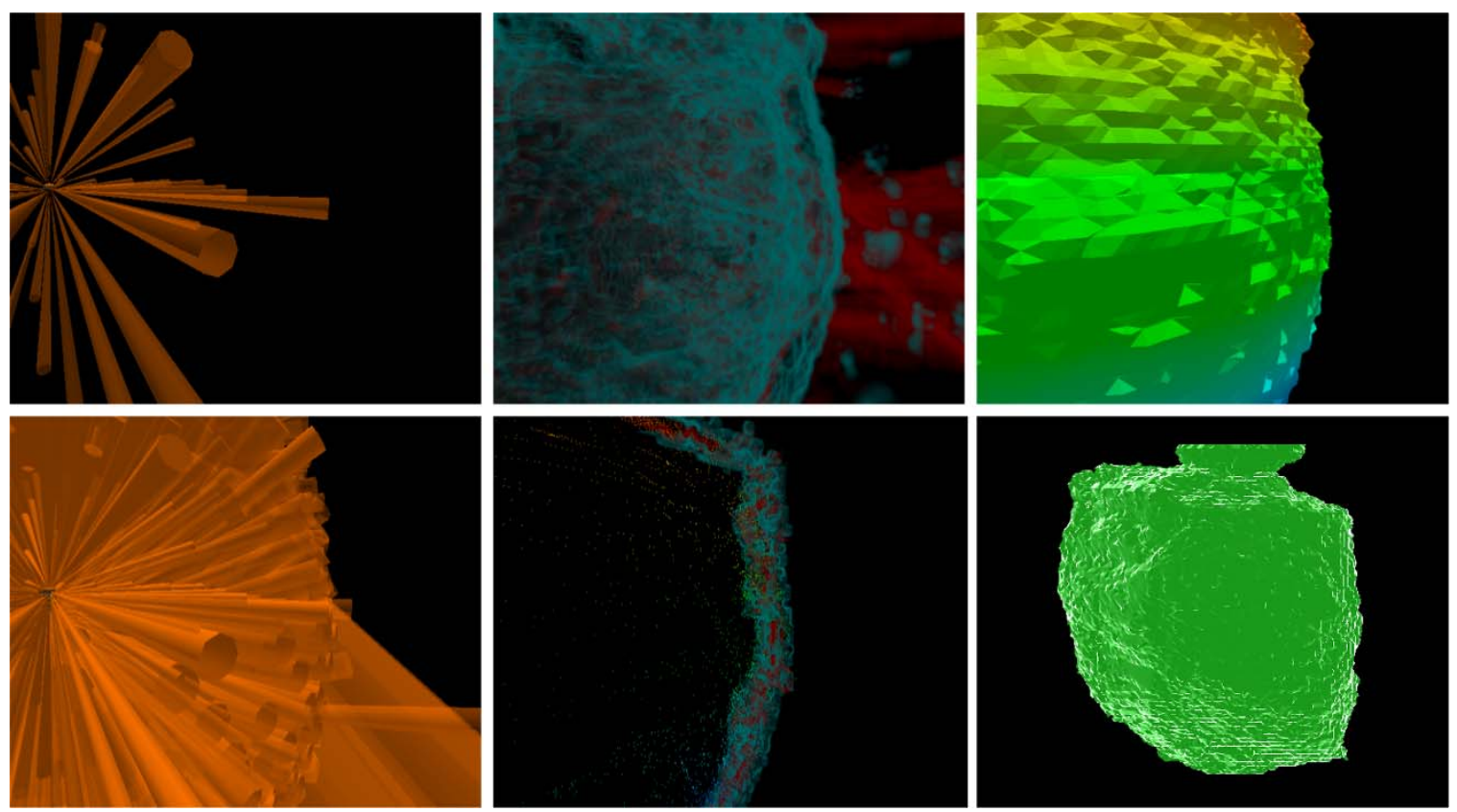

FIG. 3. This figure shows how the sonar data were processed into a map. Upper left: a rendering of the vehicle with the range values from a single ping of all the sonars (note that individual sonars often do not return range values). Lower left: by computing the vehicle's trajectory over time (using SLAM) we can combine many sonar returns into a common frame (note that only a small number of the total sonar beams are shown). Upper center: the evidence grid resulting after fusing all the sonar beams - red indicates regions of emptiness, cyan indicates regions of occupancy. Regions of high certainty, such as the entire body of the cenote which would be completely red, have been clipped out for visualization purposes. Note that there are many false "long beams", but because they are noisy uncorrelated readings their evidence does not accumulate. Bottom center: A slice through the evidence grid and through the point cloud that would result from naively plotting the ends of all the sonar beams. This shows evidence grid's ability to wash out noisy short ranges using the evidence from good ranges. Upper left: an iso-evidence surface extracted from the evidence grid using marching tetrahedra - coloration is used as a depth cue. Lower right: the entire resulting mesh of Caracol. A color image can be viewed at the website:

http://www.fieldrobotics.org/depthx/data/2007-May-maps/caracol/sequence.png

\section{RESULTS AND DISCUSSION}

\section{3-D Maps of Sistema Zacatón}

The DEPTHX vehicle explored La Pilita, Zacatón, Verde, and Caracol over the course of two field expeditions - Verde and Caracol were each explored at the very end of the field season in less than a day. During this time, the vehicle ran over 50 missions, 
ranging in duration from 10 minutes to 4 hours, collected over 30 water samples and 10 core samples from La Pilita and Zacatón, and logged an estimated 20 million sonar ranges.

The maps produced from these missions provide detailed images that reveal the geomorphic features of the cenotes (Figure 4). The cenote Zacatón is a straight vertical shaft extending below 315 meters of water depth, and is interpreted to have formed as hypogenic dissolution of the Cretaceous limestone occurred below the mapped floor (Gary and Sharp, 2006). Subsequent collapse and upward stoping results in the present morphology. Cenotes Caracol and La Pilita display a similar morphology, termed "whiskey jug cenote" from the narrow neck near the water surface of the sinkhole (Figure 4). This narrow neck is the result of calcium carbonate reprecipitation as carbon dioxide outgases from the supersaturated waters. This process has been shown to completely seal off other sinkholes in Sistema Zacatón (Gary et. al. 2006). The unique morphology of Poza Verde is hypothesized to result in similar processes that occurred when water tables were 45 meters below present day levels. The flat floor (Figure 4) of this cenote is atypical of a collapse feature, and may define this paleo-water table.

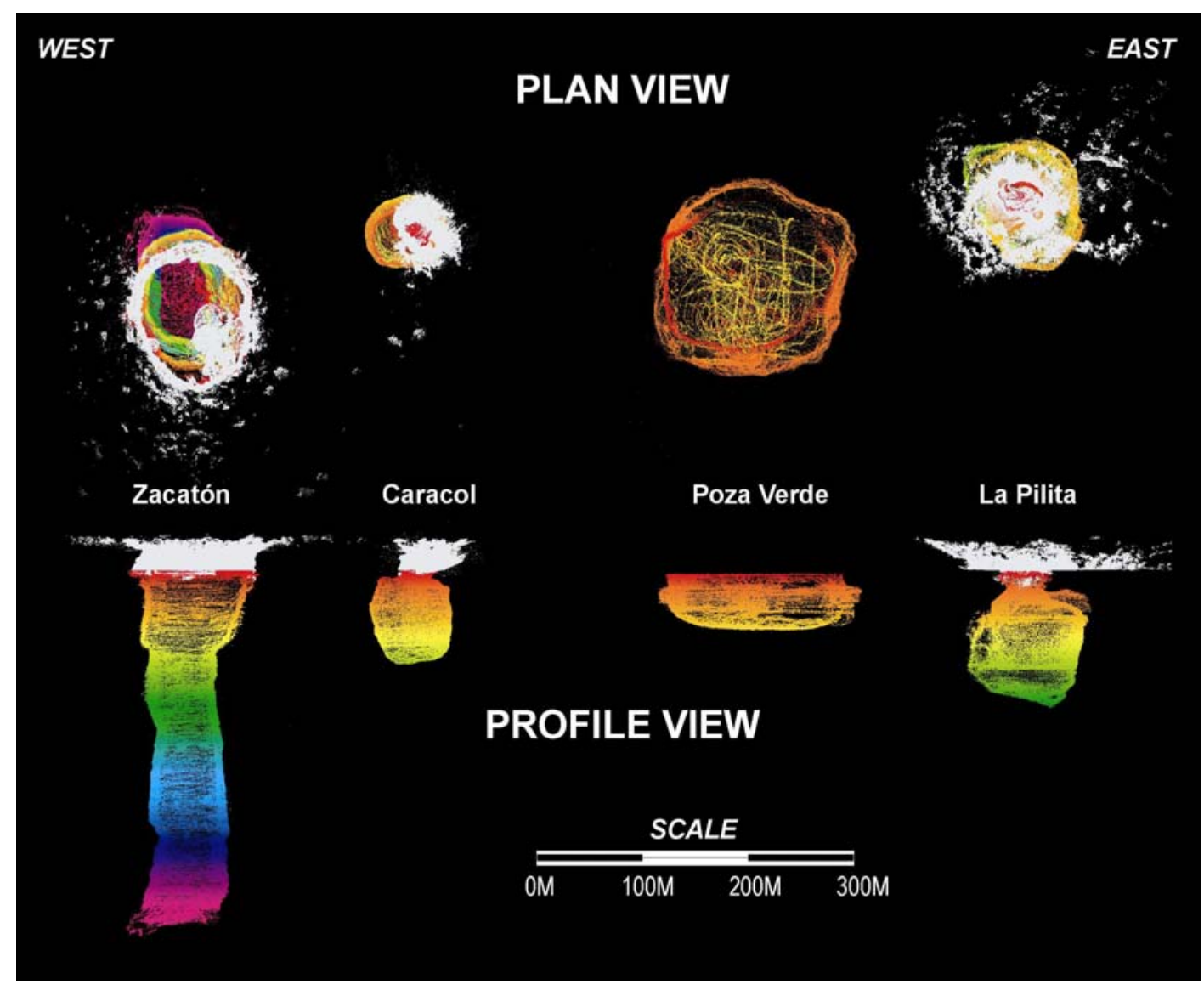

FIG. 4. Plan and profile views of the major cenotes of Sistema Zacatón as derived from surface lidar point clouds (white) and sonar point clouds (grayscale). There is no vertical exaggeration in the profile, and all features are properly georeferenced. A color image can be viewed at the website: http://www.fieldrobotics.org/depthx/data/2007-May-maps/overview.png 
One of the most powerful hydrogeologic tools of data collected by the DEPTHX vehicle is the ability to precisely measure these huge underwater void spaces. The volumes of each cenote is listed in Table A, and totals an incredible 2,763,328 cubic meters (2240 acre-feet) of water in these four caves.

Table A. The volumes of the cenotes, as computed by counting the number of evidence grid voxels with a probability of occupancy below $40 \%$.

\begin{tabular}{|l|l|}
\hline Cenote & Volume $\mathbf{( m}^{\mathbf{3}} \mathbf{)}$ \\
\hline Zacatón & $1.35 \times 10^{6}$ \\
\hline Verde & $7.51 \times 10^{5}$ \\
\hline La Pilita & $4.90 \times 10^{5}$ \\
\hline Caracol & $1.70 \times 10^{5}$ \\
\hline
\end{tabular}

\section{Geochemical Data}

Over one million data records of basic water quality parameters from the HydroTech unit were collected during the DEPTHX missions at Sistema Zacatón. Previous profile data indicated that Zacatón, Caracol, and La Pilita (deeper cenotes) were well mixed and relatively homogenous, although Zacatón had previously only been measured to a depth of 200 meters (limit of instrumentation). Poza Verde had been recognized to display stratified layers similar to a common lacustrine environment. The high density and lateral extent of DEPTHX geochemical data confirmed that all three deeper cenotes were very homogenous with respect to temperature, $\mathrm{pH}$, dissolved oxygen, and specific conductance. The waters are hydrothermal; with a constant temperature around 30 degrees $\mathrm{C}$ (figure 5) and anoxic

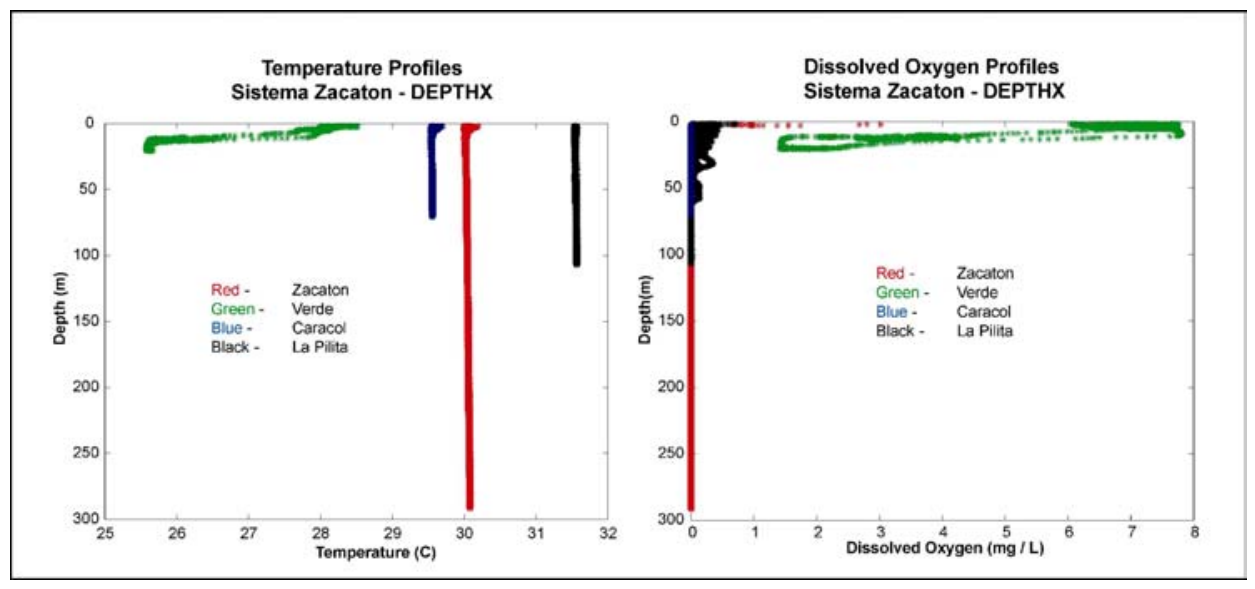

FIG. 5. Temperature and dissolved oxygen data collected from the DEPTHX vehicle show the homogenous water column for the deepest cenotes, and a different pattern for Poza Verde. 
except for shallow disoxic zones where direct diffusion from the atmosphere occurs (figure 5). Poza Verde shows distinct thermoclines and chemoclines, indicating that this body of water is somehow isolated from the deeper groundwater system accessed by the other three cenotes. This is hypothesized to occur due a thick travertine floor 45 meters below the water surface, which acts as a hydraulic boundary between deeper hydrothermal water and the perched water body of Poza Verde (Gary and Sharp, 2006).

\section{CONCLUSION}

The DEPTHX robotic exploration of Sistema Zacatón became the first autonomous instrument to map and characterize a flooded cave system. Multiple data sources have been applied to create a detailed 3D model of the cenotes, both above and below the water surface. These models aid in the geomorphic interpretation related to formation of the karst features and support previous hypothesis proposed by Gary and Sharp (2006). Additionally, the DEPTHX vehicle was able to measure basic geochemical parameters used to understand the hydrothermal nature of this karst area.

\section{ACKNOWLEDGEMENTS}

We would like to thank the NASA ASTEP program (Grant \# NNG04GC09G) for funding of the DEPTHX project. Many team members made this project successful, including: John Kerr, Dom Jonak, Ernest Franke and the Southwest Research Institute team, John Spear, Jason Sahl, Antonio Fregoso, Alonso Ramirez, and Robin Gary. Special gratitude to Alejandro Dávila who has allowed years of successful research to take place on his property, Rancho la Azufrosa. Mark Helper was critical in establishing reliable GPS positions for benchmarks that were essential in producing the maps.

A full color version of this paper can be downloaded at:

http://www.fieldrobotics.org/depthx/Gary_et_al_DEPTHX_ASCE2008.pdf

\section{REFERENCES}

am Ende, B. A. (2001). 3D mapping of underwater caves, IEEE Computer Graphics and Applications, vol. 21, no. 2, pp. 14-20.

Bakalowicz, M. (2005). Karst groundwater: a challenge for new resources. Journal of Hydrogeology 13: 148-160.

Bresenham, J. (1965). Algorithm for computer control of a digital plotter. IBM Systems Journal, 4(1):25-30.

Dissanayake, G. et al. (2001). A solution to the simultaneous localisation and map building (SLAM) problem. IEEE Transactions on Robotics \& Automation, 17(3):229241.

Fairfield, N., Kantor, G., and Wettergreen, D. (2005). Three dimensional evidence grids for SLAM in complex underwater environments. In Proc. of Intl. Symposium of Unmanned Untethered Submersible Technology.

Fairfield, N., Kantor, G., and Wettergreen, D. (2006). Towards Particle Filter SLAM with Three Dimensional Evidence Grids in a Flooded Subterranean Environment. In Proc. Of IEEE International Conference on Robotics and Automation (ICRA), Orlando, 
Florida.

Fairfield, N., Kantor, G., and Wettergreen, D. (2007). Real-time slam with octree evidence grids for exploration in underwater tunnels. Journal of Field Robotics, vol 24, issue 1-2, pages 3-21.

Gary, M.O., Halihan, T., Sharp, J.M., Mouri, S., Thorstad, J. (2006). Electrical resistivity imaging of travertine capped sinkholes: Deep lakes with lids, Geological Society of America, 2006 Philadelphia Annual Meeting Program with Abstracts, Topical Session T65, Paper No. 218-4.

Gary, M.O., Sharp, J.M.,Jr.. (2006) Volcanogenic Karstification of Sistema Zacatón, Geological Society of America Special Paper 404, p. 79-89.

Gary, M.O., Sharp, J.M.Jr., Havens, R.H., Stone, W.C. (2003). Sistema Zacatón: Identifying the Connection Between Volcanic Activity and Hypogenic Karst in a Hydrothermal Phreatic Cave System, Geo 2, Section of Cave Geology and Geography of the National Speleological Society, Volume 29, No. 3 \& 4, p. 1-14.

Gary, M.O., Stone, W.C. (2002). Creating a multi-sourced, ultra-high resolution 3-D karst aquifer model using LADAR, SONAR, and earth resistivity datasets, Proceedings of Symposium on Terrain Analysis for water resources applications, University of Texas at Austin Center of Research in Water Resources.

Gary, M.O. (2001). Los Cenotes de Rancho La Azufrosa, Association of Mexican Cave Studies Activities Newsletter, Number 24, p. 31-38.

Lorensen. W., and Cline, H. (1987). Marching Cubes: A high resolution 3D surface construction algorithm. Computer Graphics, Vol. 21, Nr. 4,

Moravec, H. and Elfes. A (1985). High resolution maps from wide angle sonar. In Proceedings of the 1985 IEEE International Conference on Robotics and Automation, pages $116-121$.

Nagihara, S., R. Goss, B. Musgrave, J. Gamel, G. Hill, and T. Bemis. (2002). Threedimensional laser scanning of speleothems in the Carlsbad Caverns: West Texas Geological Society Fall Symposium, p. 35-42.

Stone, W. C., am Ende, B. A., Wefer, F. L., and Jones,N. A. (2000). "Automated 3D Mapping of Submarine Tunnels". Proceedings, ASCE Specialty Conference: Robotics 2000, W.C. Stone, Ed., American Society of Civil Engineers, 1801 Alexander Bell Drive, Reston, Virginia 20191-4400.

Stone, W.C. (2007). Design and Deployment of a 3D Autonomous Subterranean Submarine Exploration Vehicle", Proceedings UUST07, Conference on Un-manned, Un-tethered Submersable Technology, Durham, NH, August 20-22, 2007.

Veni, G. (1999). A geomorphological strategy for conducting environmental impact assessments in karst areas. Geomorphology 31: 151-180.

White, W.B. (2002). Karst hydrology: recent developments and open questions. Engineering Geology 65: 85-105. 